\title{
Recombinant yeast and human cells as screening tools to search for antibacterial agents targeting the transcription termination factor Rho
}

\author{
Kévin Moreau ${ }^{1} \cdot$ Justine Surand $^{1} \cdot$ Aurélia Le Dantec $^{1} \cdot$ Christine Mosrin-Huaman $^{1} \cdot$ Alain Legrand $^{2}$. \\ A. Rachid Rahmouni ${ }^{1}$
}

Received: 31 August 2017 / Revised: 24 October 2017 / Accepted: 31 October 2017 / Published online: 25 January 2018

(c) The Author(s) 2018. This article is published with open access

\begin{abstract}
The alarming issue of antibiotic resistance expansion requires a continuous search for new and efficient antibacterial agents. Here we describe the design of new tools to screen for target-specific inhibitors of the bacterial Rho factor directly inside eukaryotic cells. Rho factor is a global regulator of gene expression which is essential to most bacteria, especially Gramnegative. Since Rho has no functional or structural homolog in eukaryotes, it constitutes a valuable and well known bacterial target as evidenced by its inhibition by the natural antibiotic, Bicyclomycin. Our screening tools are based on perturbation of mRNA processing and packaging reactions in the nucleus of eukaryotic cells by the RNA-dependent helicase/translocase activity of bacterial Rho factor leading to a growth defect phenotype. In this approach, any compound that impedes Rho activity should restore growth to yeast or human cells expressing Rho protein, providing valuable means to screen for targetspecific antibacterial agents within the environment of a eukaryotic cell. The yeast tool expressing $E$. coli Rho factor was validated using Bicyclomycin as the control antibacterial agent. The validation of the screening tool was further extended with a stable human cell line expressing Rho factor conditionally. Finally, we show that Rho factors from different bacterial pathogens can also be designed as yeast-based screening tools which can reveal subtle variations in the functional features of the proteins.
\end{abstract}

\section{Introduction}

The continuous emergence and expansion of antibiotic resistant bacteria constitutes a serious threat for public health. This concern leads to an urgent requirement for the discovery of new and more efficient antibacterial agents, preferentially compounds that are not susceptible to existing mechanisms of resistance [1,2]. Among the innovative drug discovery approaches, one strategy to discover new antibiotics relies on in vitro biochemical screening of large collections of synthetic or natural compounds searching for binders and/or inhibitors of well-known and relevant

A. Rachid Rahmouni

rahmouni@cnrs-orleans.fr

1 Centre de Biophysique Moléculaire, UPR 4301 du CNRS, Rue Charles Sadron, 45071 Orléans, France

2 University of Orléans, Pole Universitaire Centre Val de Loire, Orléans, France bacterial targets [3]. In contrast to the older whole-cell screening methods that seek inhibition of bacterial cell growth or cell killing, the in vitro biochemical screening strategies avoid the hazardous manipulations of pathogens and hence, they are more amenable to high throughput screening methodologies (HTS). In addition, the interrogation of very large chemical libraries by HTS can be very advantageous by revealing multiple compounds acting on the same bacterial target and which can have additive or synergistic effects when used in combination [4]. However, such target-oriented in vitro screening approach for drug discovery can be very laborious and time-consuming. First, it requires the production and purification of sufficient amounts of the bacterial target to be analyzed, usually a macromolecule or a multicomponent complex. Following biochemical characterization, the approach needs the setup of an in vitro assay to measure the inhibition of the target activity. Within the context of HTS measurements, the assay should be easy-to-use and cost-effective to allow the interrogation of large chemical collections as well as reiterative screening for downstream explorations of 
structure-activity relationship to improve efficacy and optimize pharmacology of potential lead compounds.

Rho factor, which belongs to the family of ring-shaped homo-hexameric helicases, is a global regulator involved in about $50 \%$ of the transcription termination events in bacteria such as in the model micro-organism Escherichia coli. In addition to its involvement in the regulation of gene expression and the control of pervasive transcription [5-7], Rho plays also a major role in a number of other physiological processes including the maintenance of bacterial genome integrity by silencing foreign DNA expression and preventing the formation of transcription-driven R-loops [8-10]. These important functions explain why Rho is essential for cell growth and is highly conserved across most bacterial phyla, especially in Gram-negative bacteria [11]. Interestingly, the absence of structural and functional homologues in eukaryotes makes Rho a very attractive target for antibacterial drug discovery. Indeed, Rho is the target of the natural product Bicyclomycin (BCM) which was isolated in 1972 from two different Streptomyces strains and which is an effective antibiotic against Gramnegative bacteria (Reviewed in [12]). BCM exerts its action by inhibiting the ATPase activity of Rho which is required for its RNA-dependent helicase/translocase functioning [13-15]. However, BCM did not have a large pharmaceutical success because it is poorly absorbed when taken through the gastrointestinal tract and thus, it is a weak antibiotic for systemic infections. Other limitations of BCM as an antibiotic stem from the absence of effective activity towards some bacterial pathogens including Grampositive strains, as well as the occurrence of mutationinduced resistant cells (reviewed in [12]). Nevertheless, Rho remains a valuable bacterial target for drug discovery as highlighted by a recent study showing that its depletion from Mycobacterium tuberculosis leads to rapid cell death both for in vitro cultures and in vivo infections in mice [16].

We took advantage of the functional features of Rho to implement an innovative eukaryotic cell-based approach that can be used as a tool to screen for new antibacterial compounds targeting Rho and to optimize their pharmaceutical efficiency through reiterative screening of lead derivatives. The method is based on perturbation of the cotranscriptional mRNA processing and packaging reactions in the yeast $S$. cerevisiae and human cells by the RNAdependent helicase/translocase activity of the bacterial Rho factor. The heterologous expression of Rho and its localization in the nucleus of the eukaryotic cells lead to a growth defect phenotype that can be easily monitored. Thus, any compound that impedes Rho activity will restore cell growth, providing a valuable tool to search for efficient target-specific antibacterial agents within the environment of a eukaryotic cell. We report the design and validation of the approach in a microplate-based system using yeast $S$. cerevisiae strain expressing $E$. coli Rho and with BCM as the control Rho-specific antibacterial agent. The validation of the approach was extended further using a recombinant human cell line expressing the Rho factor conditionally. Yeast-based screening tools were also constructed with Rho factors originating from different bacterial pathogens.

\section{Materials and methods}

\section{Yeast cells and plasmids}

S. cerevisiae yeast cells (BMA41 MAT $\alpha$ ) were grown according to standard procedures at $25^{\circ} \mathrm{C}$ in synthetic medium lacking some nucleobases and amino acids as necessary for selection and with glucose $(2 \%)$ as the carbon source. The cell growth was monitored by measuring the optical density (OD) at $595 \mathrm{~nm}$. The construction of the yeast plasmids expressing $E$. coli Rho with or without a Nuclear Localization Signal (NLS), Rho-NLS or Rho$\Delta$ NLS respectively, under the control of the Doxycyclinerepressed promoter $\left(\mathrm{TetO}_{7}\right)$ was previously described [17]. Briefly, the Rho open reading frame was amplified by PCR from E. coli genomic DNA using appropriate primers having or not the SV40 NLS sequence and designed to generate NotI restriction sites at both ends. The PCR fragments were trimmed with Not I and inserted into the pCM185 (TRP1 Marker) or pCM189 (URA3 Marker) vectors which harbor the constitutively expressed transactivator, tTA [18]. The same cloning process was utilized to construct the plasmids expressing Rho factors from different pathogens following PCR amplification of the open reading frames using genomic DNAs obtained from CIRM (Centre International de Resources Microbiennes, Centre INRA Val de Loire, France). The appropriate plasmid clones were selected after sequencing and verification of protein expression by Western blot. The expression of Rho is repressed by growing the yeast cell in the presence of Doxycycline (Doxy) at the concentration of $5 \mu \mathrm{g} / \mathrm{ml}$ and its omission in the growth medium allowed maximum expression.

For the drop test experiments, ten-fold serial dilutions of yeast cultures were spotted on agar plates with or without Doxy and photographed after 3 days growth at $25^{\circ} \mathrm{C}$. For the Kirby-Bauer test, agar plates with or without $5 \mu \mathrm{g} / \mathrm{ml}$ Doxy were seeded with a lawn of $S$. cerevisiae bearing the Rho expression plasmid. Paper disks were dispensed on the agar surface and spotted with $7 \mu$ l of a solution of BCM at the indicated concentration or $7 \mu \mathrm{l}$ of water for the control. The plates were incubated at $25^{\circ} \mathrm{C}$ for 3 days before taking the picture. 


\section{Yeast growth in 96-well microplate}

The yeast cells transformed with the appropriate Rho expression plasmid were pre-grown overnight under repressing conditions with $5 \mu \mathrm{g} / \mathrm{ml}$ Doxy at $25{ }^{\circ} \mathrm{C}$ in a shaker $(255 \mathrm{rpm})$. Three milliliter medium were seeded with the culture and grown for $5 \mathrm{~h}$ with $1 \mu \mathrm{g} / \mathrm{ml}$ Doxy at $25^{\circ} \mathrm{C}$ in a shaker $(255 \mathrm{rpm})$. At this point, the OD of the culture at $595 \mathrm{~nm}$ is usually between 0.7 and 0.8 . The cells were then centrifuged for $10 \mathrm{~min}$ and washed three to four times with $5 \mathrm{ml}$ of fresh medium lacking Doxy. The cells were resuspended in the medium at a density of $1 \mathrm{OD}$ (equivalent to $2 \times 10^{7} \mathrm{cell} / \mathrm{ml}$ ) and a final dilution at $1 / 20$ was made. Two hundred microliter of the cell suspension were dispensed in each well of a 96-well flat bottom microplate with the addition of Doxy and BCM when needed. Each growth condition sample was performed in triplicate. The microplate was covered with a lid and introduced into a TECAN incubator for growth monitoring with the following parameters: temperature $25^{\circ} \mathrm{C}, 24$ cycles of absorbance monitoring at $595 \mathrm{~nm}$ with $1 \mathrm{~h}$ interval, shaking for $1 \mathrm{~min}$ at 432 rpm each $10 \mathrm{~min}$. The data, time points versus OD, were processed with excel software.

\section{Generation of mammalian cell lines expressing Rho}

For the construction of the mammalian plasmids, the open reading frames of Rho-NLS or Rho- $\Delta$ NLS were amplified by PCR from the yeast plasmids using appropriate primers designed to generate HindIII and XhoI restriction sites at the upstream and downstream ends of the amplicons, respectively. After restriction cutting, the DNA fragments were inserted between the HindIII and XhoI sites within the polylinker of the pIND mammalian expression vector (Invitrogen). The integrity of the resulting plasmids (pIND-Rho-NLS and pIND-Rho- $\Delta$ NLS) was verified by sequencing.

EcR 293 cells, derived from HEK 293 cells by incorporation of the regulatory ecdysone-inducible mammalian expression system within the vector $\mathrm{pVgRXR}$ (Invitrogen), were transfected with $30.4 \mu \mathrm{l}$ of PEI $10 \mu \mathrm{M}$ (SIGMA Aldrich) in sterile $\mathrm{H}_{2} \mathrm{O}$ mixed with $4.5 \mu \mathrm{g}$ of pIND-RhoNLS or the pIND-Rho- $\Delta$ NLS plasmid DNA diluted in $1 \mathrm{ml}$ of physiological serum. After $20 \mathrm{~min}$ incubation at room temperature, the plasmid/PEI mix was directly added drop by drop into the culture wells containing cells in DMEM Fetal Bovine Serum (FBS) free medium. Cells were incubated for $2 \mathrm{~h}$ at $37^{\circ} \mathrm{C}\left(5 \% \mathrm{CO}_{2}\right)$, then the medium was replaced by complete DMEM AQmedia ${ }^{\mathrm{TM}}$ (SIGMA Aldrich ref: D0819) culture medium containing 1\% penicillin/streptomycin (SIGMA Aldrich ref: P0781), $4 \mu \mathrm{l}$ of Zeocin (Invitrogen ref: ant-zn-1) at $100 \mathrm{mg} / \mathrm{ml}$ and $10 \%$ FBS (SIGMA Aldrich ref: F7524). Following transfection, stable transfectants were selected by dilution cloning based on growth in the presence of G418 at $800 \mu \mathrm{g} / \mathrm{ml}$ (resistance gene in pIND) and zeocin at $400 \mu \mathrm{g} / \mathrm{ml}$ (resistance gene in pVgRXR). The growing clones were first verified by PCRamplicon sequencing then confirmed by Western blot analyses of Rho expression induced after addition of Ponasterone A (Sigma Aldrich ref: P3490) into the medium at $5 \mu \mathrm{g} / \mathrm{ml}$. Among many positive clones, appropriate cell lines exhibiting the best repression and inducibility of Rho were chosen. Aliquots of the cell lines were frozen for cryopreservation in liquid nitrogen and a new aliquot was used after each 10 passages. For each passage, confluent cells were rinsed with phosphate-buffered saline (PBS) $\mathrm{pH}$ 7.4. After cell detachment using trypsin, the cell suspension was used to seed new culture flasks at a 1/10 split ratio.

\section{Mammalian cell viability assay}

Rho-expressing EcR 293 cell lines were seeded into a 96well plate at 7.000 cells per well in $100 \mu$ l of DMEM $\mathrm{AQmedia}^{\mathrm{TM}}$ culture medium. Following cells adhesion, Rho induction was performed by replacing the culture medium by fresh medium containing Ponasterone A at $5 \mu \mathrm{g} / \mathrm{ml}$. When needed, BCM was directly added to the wells at different concentrations $(10,20$ or $50 \mu \mathrm{g} / \mathrm{ml}$, final concentration) at the $12 \mathrm{~h}$ growth time point. Cells viability was assessed at each time point by using PrestoBlue ${ }^{\mathrm{TM}}$ Viability Reagent (life technologies ref: A13262) according to the manufacturer's instructions. Briefly, $10 \mu \mathrm{l}$ of the reagent were added directly to the culture medium in each well. Following $2 \mathrm{~h}$ incubation $\left(37^{\circ} \mathrm{C}, 5 \% \mathrm{CO}_{2}\right)$, the plate was shaken $5 \mathrm{~min}$ on a plate shaker then read with a Victor $3 \mathrm{~V}$ plate reader (PerkinElmer). Shortly after reading the plate, pictures were taken with a 16 million pixel camera by pipetting the colored media from the wells and pouring them into a new 96-well plate.

\section{Western blotting}

Total proteins in whole-cell extracts were obtained as described [19], then resolved on SDS-10\% polyacrylamide electrophoresis gels and analyzed by Western blotting according to standard procedures. The Rho protein was detected with rabbit anti-Rho antibodies (a custom preparation from Eurogentec, Belgium). Secondary antibodies (goat anti-rabbit-HRP IgG, Promega) were used and visualization on $\mathrm{X}$-ray films was performed following enhanced chemiluminescence (Clarity Western ECL Substrate from Biorad).

\section{Structure and multi-alignment study}

Structure visualization was performed using The PyMOL Molecular Graphics System, Version 1.7.2.1 Schrödinger, 


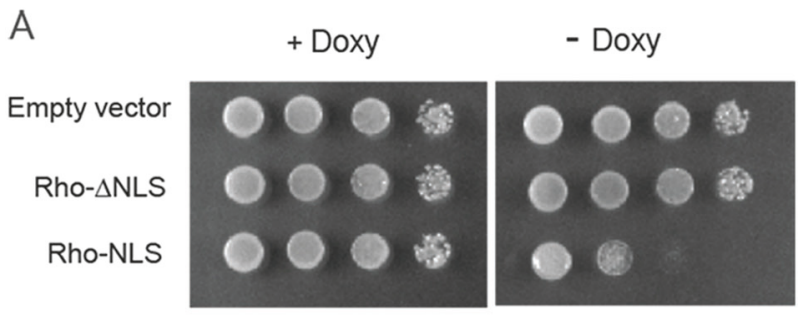

B
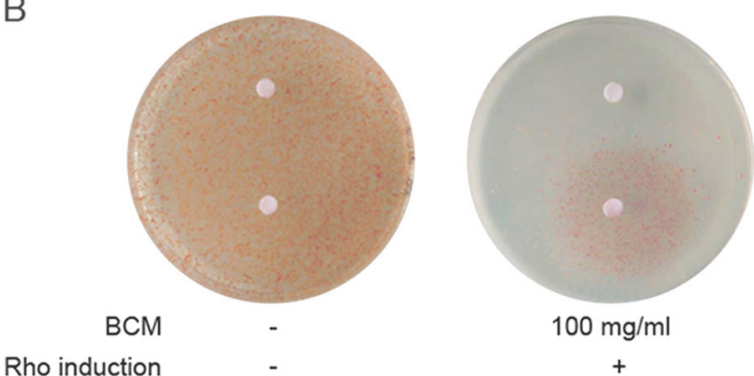

Rho induction

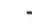

C

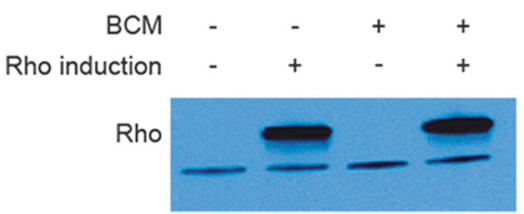

Fig. 1 Rho expression in the nucleus of yeast cells induces a growth defect. a Ten-fold serial dilutions of yeast cells transformed with the empty vector or the indicated plasmids expressing Rho-NLS or Rho$\Delta \mathrm{NLS}$ were spotted on agar plates in the presence or absence of $5 \mu \mathrm{g} / \mathrm{ml}$ of Doxy and grown at $25^{\circ} \mathrm{C}$ for 3 days. b Kirby-Bauer disk diffusion test. Agar plates with or without $5 \mu \mathrm{g} / \mathrm{ml}$ Doxy were seeded with a lawn of $S$. cerevisiae bearing the Rho-NLS expression plasmid. Paper disks were dispensed on the agar surface and spotted with $7 \mu$ of a solution at $100 \mathrm{mg} / \mathrm{ml}$ of Bicyclomycin (BCM, bottom disk) or $7 \mu \mathrm{l}$ of water (top disk) for the control. Pictures were taken after 3 days incubation at $25^{\circ}$ C. c Western blot using anti-Rho antibodies showing Rho expression upon induction by omission of Doxy in the growth medium in the absence or presence of $100 \mu \mathrm{g} / \mathrm{ml}$ of BCM. The lower band corresponds to a yeast protein cross-reacting non-specifically with the anti-Rho antibodies

LLC. Structural data were downloaded from PDB (accession number 1XPU). Multiple sequence alignments were done using the EBI online tool Clustal Omega with default parameters.

\section{Results}

\section{The activity of $\mathrm{E}$. coli Rho factor in the nucleus of S. cerevisiae impedes cell growth}

Bacterial Rho factor functions as a powerful helicase/ translocase that uses energy from ATP hydrolysis to track along an RNA chain and can melt nucleic acid base pairing or disrupt RNA-protein complexes present in its path $[20,21]$. We have shown previously that the activity of Rho in the yeast nucleus impairs the co-transcriptional recruitment of mRNA processing and packaging factors yielding mRNAs that are aberrantly processed or depleted of some crucial export proteins. Such defective mRNAs are targeted and eliminated by the nuclear quality control system coupled to the RNA degradation machinery, a process that hinders cell fitness and growth [22-24]. As shown in Fig. 1a with drop tests, ectopic expression in S. cerevisiae of $E$. coli Rho factor harboring a Nuclear Localization Signal (RhoNLS) at its C-terminus leads to a growth defect phenotype. Rho is expressed under the control of a Doxycyclinrepressed $\mathrm{TetO}_{7}$ promoter within a centromeric plasmid bearing the constitutively expressed trans-activator (tTA). The growth defect is not observed when the ectopically produced Rho is no longer addressed to the cell nucleus by the localization signal (Rho- $\Delta$ NLS construct). Interestingly, a similar relief of growth defect is achieved by inhibiting Rho activity with the antibiotic BCM as observed by the Kirby-Bauer disk diffusion test (Fig. 1b). The revival of yeast cells expressing Rho in a zone surrounding the paper disk containing $\mathrm{BCM}$ is an indication regarding the target specificity of the antibiotic and the absence of any obvious toxicity towards the eukaryotic host cells. As shown by Western blot, the suppression of the growth defect by BCM is clearly linked to the inhibition of Rho activity and not to a potential shutdown of the protein production (Fig. 1c).

\section{Microplate-based measurements of Rho-induced growth defect in yeast}

To adapt our approach to high-throughput screening conditions, we monitored the Rho-induced growth defect in liquid medium using 96-well microplates with $200 \mu \mathrm{l}$ cultures. The growth measurements were conducted by recording the OD of the cultures at $595 \mathrm{~nm}$ every hour over a period of $24 \mathrm{~h}$ with vigorous shaking at $25^{\circ} \mathrm{C}$ in a TECAN incubator (Fig. 2). The control growth curve of yeast cells containing an empty vector reached the stationary phase plateau at $1.2 \mathrm{OD}$ after 18 to $20 \mathrm{~h}$ incubation under both repressing (+Doxy) and inducing (-Doxy) conditions. A similar growth curve profile was observed for the yeast cells harboring the Rho expression plasmid and grown under repressing conditions. In contrast, the growth curve of yeast cells containing the Rho expression plasmid remained flat under inducing conditions, reaching only 0.2 OD after $20 \mathrm{~h}$ of growth. In agreement with the disk diffusion test results, the presence of BCM in the medium restores growth to the cells expressing Rho with a curve profile rising up to $0.8 \mathrm{OD}$ when the concentration of the antibiotic was raised to $200 \mu \mathrm{g} / \mathrm{ml}$. These growth profiles indicate that the rescue is not complete, probably due to a limited intracellular availability of the Rho inhibitor (see discussion). However, the mitigation of Rho-induced 


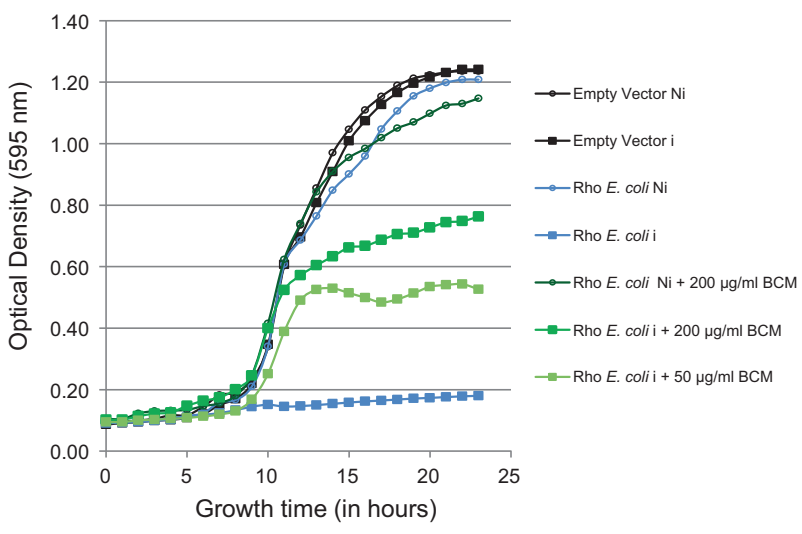

Fig. 2 Growth curves of yeast cells harboring the empty plasmid or the plasmid expressing $E$. coli Rho-NLS. The growth was monitored in a TECAN incubator with $200 \mu \mathrm{l}$ cultures in 96-well microplates under inducing (i) or non-inducing (Ni) conditions. For the relevant cultures, $\mathrm{BCM}$, at the indicated final concentration, was added in the medium at the start of growth

growth defect by BCM became clearly apparent after only 10 to $12 \mathrm{~h}$ growth. Thus, within the context of an HTS screen, it is possible to detect within this time interval the inhibitory effect of a potential compound targeting Rho activity.

\section{Recombinant human cell line as Rho-based screening tool}

Conceivably, potential inhibitors of Rho activity may escape detection during a screening process with the yeast tool if they have an antifungal activity that kills the cells. To circumvent this possible limitation, we developed a recombinant human cell line as a complementary Rho-based screening tool. DNA encoding the open reading frame of Rho-NLS or Rho- $\Delta$ NLS was shuffled from the yeast plasmids into the pIND mammalian expression vector and the resulting constructs were transfected into EcR 293 cells (HEK 293 cells derivatives which were stably transformed with the regulatory vector $\mathrm{pVgRXR}$ ) [25]. Resistance to Zeocin and G418 was used to select for potential positive clones. Stable clonal cell lines were established and the induction of Rho expression by Ponasterone was confirmed by Western blot analyses as described in Materials and Methods and shown in Fig. 3a. The selected cell lines showed a tight regulation of the expression system with the appearance of Rho protein $6 \mathrm{~h}$ following induction (Fig. 3b).

To evaluate the effect of Rho expression on cell proliferation, the growth of the selected cell lines was monitored visually on 6-well plates over a period of 7 days. In the absence of Rho expression, the cells grew to at least $90 \%$ confluence during the 7 days period, covering nearly the whole surface of the well (Fig. 3c, lane 1). The
A

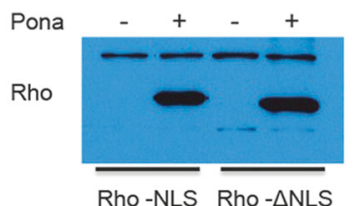

B

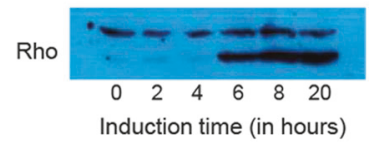

C

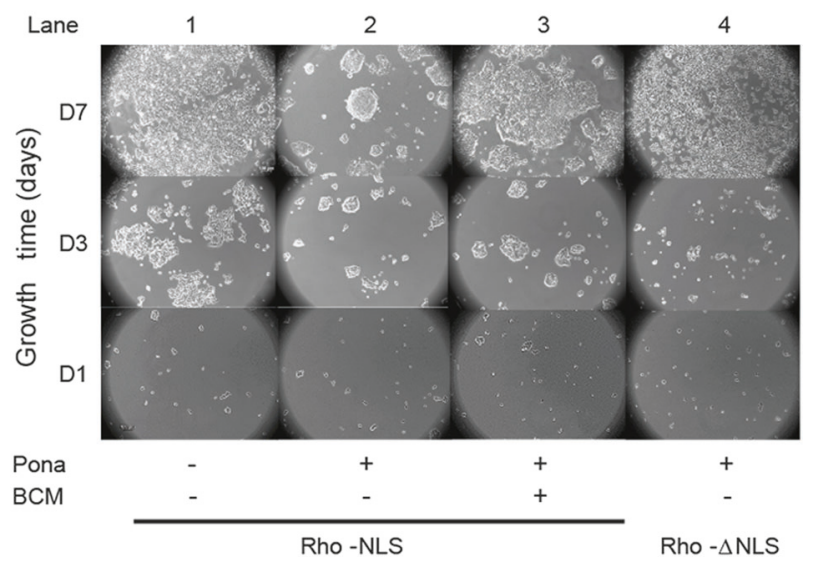

Fig. 3 Rho expression in EcR 293 cell lines induces a cytostatic effect. a Western blot showing the conditional expression of Rho-NLS and Rho- $\Delta$ NLS by the recombinant EcR 293 cell lines. Pona stands for the inducer Ponasterone which was added to the cell culture medium at $5 \mu \mathrm{M}$. The upper band corresponds to a protein cross-reacting nonspecifically with the anti-Rho antibodies. b Western blot showing the expression of Rho-NLS as a function of time. c Photograph showing the growth over 7 days of the recombinant EcR 293 cell lines expressing conditionally Rho-NLS or Rho- $\Delta$ NLS. The addition of $\mathrm{BCM}$ to the relevant culture at $50 \mu \mathrm{g} / \mathrm{ml}$ final concentration was done $12 \mathrm{~h}$ after cells seeding

induction of Rho-NLS expression by Ponasterone had a dramatic effect on growth with the cells reaching only 25-30\% confluence after the 7 days incubation (Fig. 3c, lane 2). This cytostatic effect was not observed with the cell line expressing the Rho derivative lacking the NLS (Rho$\Delta$ NLS, lane 4), emphasizing the need for nuclear accumulation of the bacterial factor to impair the growth of the eukaryotic cell. Importantly, the addition of BCM to the culture medium ( $12 \mathrm{~h}$ after cell seeding) alleviates the cytostatic effect of Rho-NLS expression with the cells reaching nearly $80 \%$ confluence after the 7 days of growth (Fig. 3c, lane 3). These results show that Rho expression in the nucleus of human cells induces a similar growth defect than in yeast.

\section{Adaptation of the Rho-based human cell line tool to HTS conditions}

To adjust the new tool to high-throughput screening conditions, the effect of Rho expression on cell proliferation was assessed quantitatively using the resazurin-based PrestoBlue ${ }^{\circledR}$ Cell Viability Reagent that measures the reducing 
environment of viable cells. In the presence of live cells, the color of the reagent turns from blue to red whose intensity can be measured by absorbance-based plate readers. The cell viability measurements were processed at 12, 24, 72, and $96 \mathrm{~h}$ after cell seeding. As shown by the plate photograph in Fig. $4 \mathrm{a}$, the detection by the reagent is in complete agreement with the visual observation of the cell growth. In the absence of Rho-NLS expression or with the expression of Rho- $\Delta$ NLS, a switch to red color was observed over the growth period. In contrast, the induction of Rho-NLS expression did not show significant color change over time, an indication for low cell density. Again, the effect of BCM in rescuing the cell proliferation is clearly seen by the color indications in the wells. The absorbance values of the samples taken at each time point were plotted to reveal the growth curve profiles (Fig. 4b). The graph reveals that the effect of Rho inhibition can be assessed as early as $36 \mathrm{~h}$ after compound addition ( $48 \mathrm{~h}$ after cell seeding) with a larger confidence at the $72 \mathrm{~h}$ time point of growth where there is more than twofold difference in the level of cell viability between the samples with and without BCM addition. Together, these results show that the human cell tool is readily appropriate for large scale screening of compound libraries to search for potential inhibitors of bacterial Rho activity.

\section{Yeast growth defect induced by Rho proteins originating from different bacterial pathogens}

In general, the amino acids involved in Rho functional features such as RNA binding and ATP hydrolysis are relatively conserved across bacterial phyla. However, some variations in other functionally important motifs that mediate the RNA-dependent helicase/translocase activity have been observed among Rho proteins from different bacterial species ([11] and references cited therein). Having this in mind, we wanted to assess the extent to which the yeastbased screening tool can be generalized to other bacteria and especially some clinically relevant pathogenic strains. To this end, we cloned Rho proteins of A. baumannii, $E$. coli ESBL, E. cloacae, E. faecalis, P. aeruginosa, and $S$. enterica in the yeast centromeric expression vector with the NLS at their C-terminus. After transformation into $S$. cerevisiae, the expression of the different Rho proteins was verified by Western blot (Fig. 5a). Although we did not perform quantitative assessment of the expression level for each Rho protein, some Rho species appear to be less expressed compared to the expression level of E. coli Rho. Notably, the Western blot reveals lower accumulations for Rho proteins from A. baumannii, E. faecalis, and $P$. aeruginosa.

The effect of the different Rho proteins on the growth of $S$. cerevisiae was first monitored by serial dilutions on agar

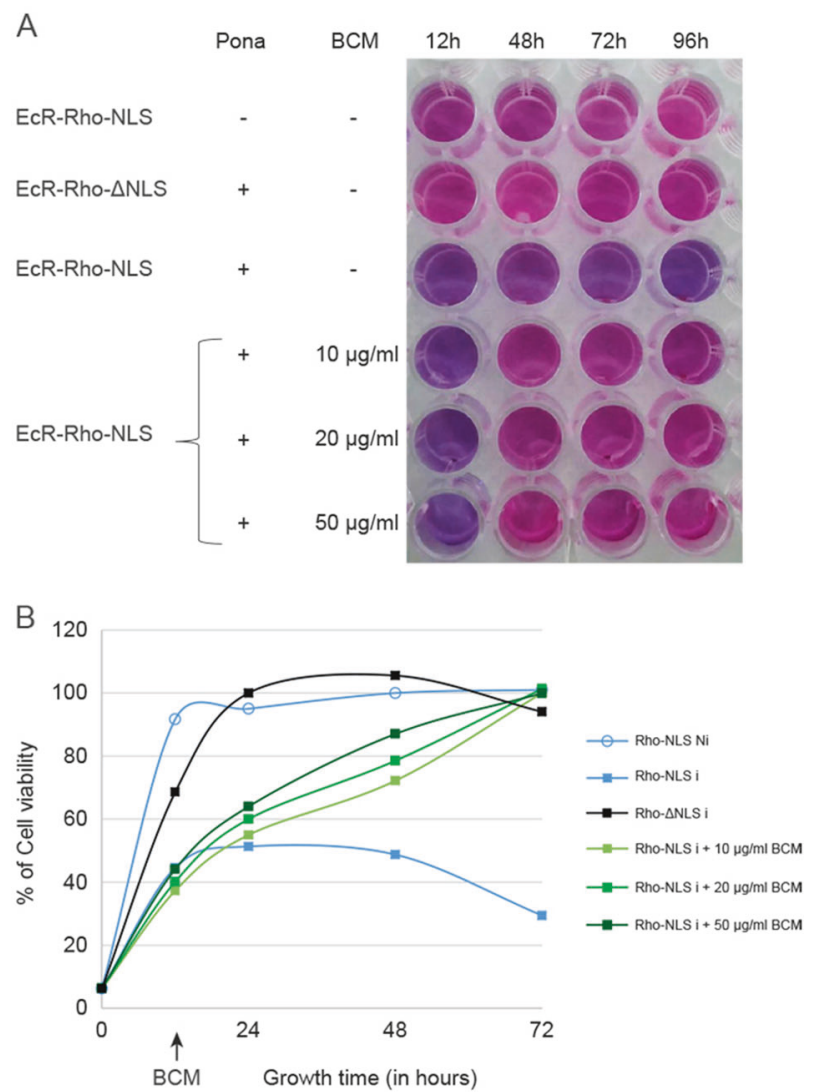

Fig. 4 Quantitative measurements of mammalian cells viability following Rho expression. a Picture of a part of a 96-well plate showing the colored media resulting from the reaction of PrestoBlue ${ }^{\mathrm{TM}}$ Viability Reagent. BCM was added to the media of the relevant culture wells at the indicated final concentration $12 \mathrm{~h}$ after cell seeding. b Graphs showing the growth curves obtained by reading the 96-well plate with a Victor $3 \mathrm{~V}$ plate reader following revelation by PrestoBlue $^{\mathrm{TM}}$. (Ni) stands for non-induced and (i) for induced

plates in the presence or absence of the effector, Doxy (Fig. 5b). Under inducing conditions (-Doxy), the growth of the yeast cells expressing Rho proteins was severely reduced. However, the growth defect seems to be notably less pronounced with the $P$. aeruginosa Rho protein. This trend was also observed under HTS-like growth conditions by monitoring the growth in liquid medium using 96-well microplates with $200 \mu \mathrm{l}$ cultures (Fig. 6).

The moderate effect of $P$. aeruginosa Rho on yeast growth may imply a less proficient helicase/translocase activity of the protein. This possibility was explored by comparing the Rho sequence of $P$. aeruginosa to that of E. coli (Fig. 7a). Interestingly, the sequence alignment shows a substitution of an important and highly conserved amino acid in the ATP binding pocket for $P$. aeruginosa Rho (Met-186-Ile). Indeed, as illustrated in Fig. 7b, previous structural studies of $E$. coli Rho have shown that the side chains of two conserved amino acids, Met-186 and Phe-355, are responsible for sandwiching the adenine 


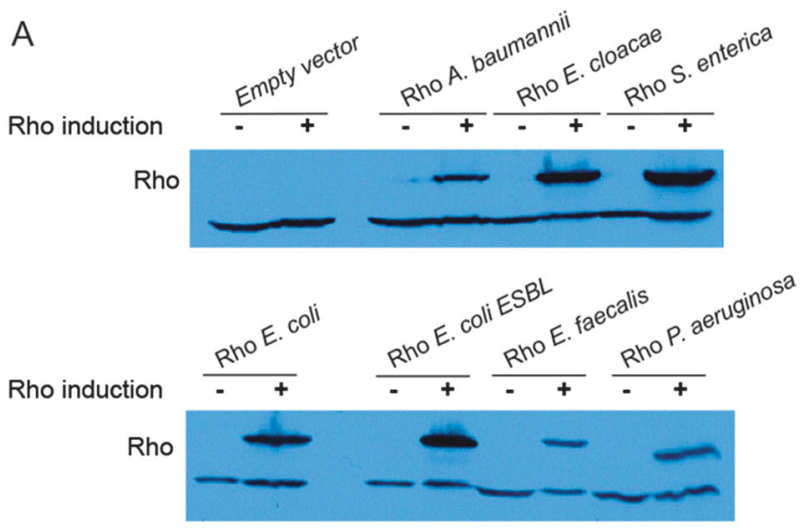

B
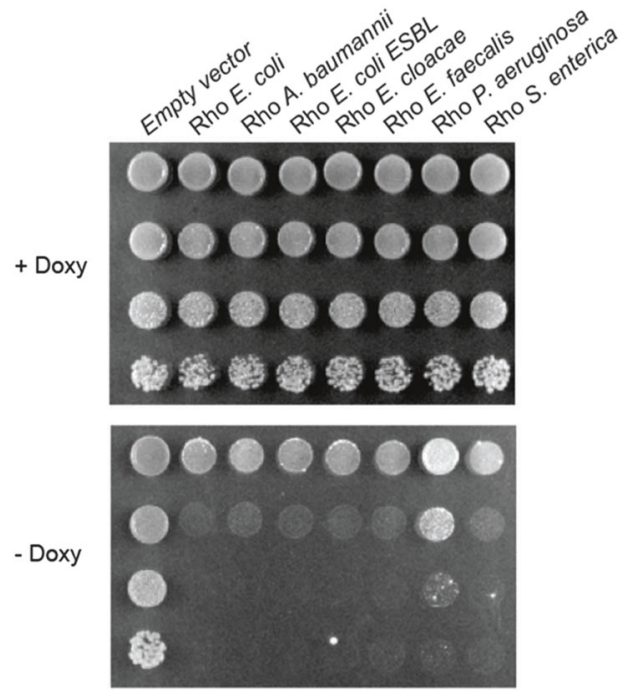

Fig. 5 Yeast growth defect induced by Rho proteins from different bacterial pathogens. a Western blot showing the conditional expression in yeast of Rho proteins from the indicated bacterial strains. $\mathbf{b}$ Ten-fold serial dilutions of cells transformed with the empty vector or the plasmid expressing the indicated Rho variants having an NLS at their C-terminus. The cells were spotted on agar plates with or without $5 \mu \mathrm{g} / \mathrm{ml}$ Doxy and incubated at $25^{\circ} \mathrm{C}$ for 3 days

moiety of bound ATP [26]. Thus, one possibility could be that lower binding affinity of ATP may decrease the rate of its hydrolysis which in turn will weaken the strength and/or the pace of Rho translocation along the RNA chain. Such scenario could explain a less efficient interference in the yeast mRNA processing and packaging reactions with ensuing modest impairment to the cell fitness.

\section{Discussion}

The development and spread of antibiotic resistance in bacteria is a global threat to public health. One useful way to tackle this concern resides in the search for new drugs that inhibit the activity of well-known and relevant bacterial

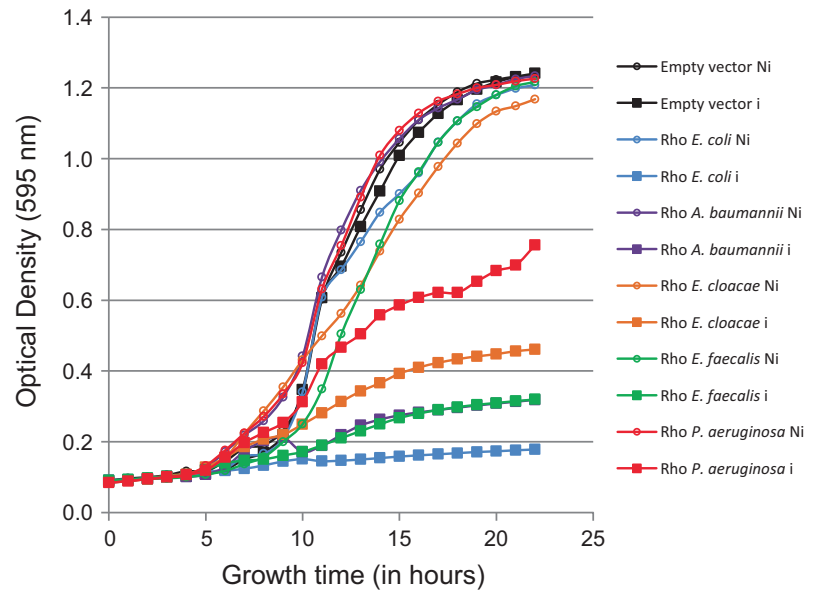

Fig. 6 Growth curves of yeast cells harboring the empty plasmid or the plasmid expressing Rho-NLS proteins originating from the indicated bacterial strains. The growth was monitored in a 96-well plate as indicated in Fig. 2. (Ni) stands for non-induced and (i) for induced

molecular targets. Hence, this requires the design of original methodologies and tools to screen, at high throughput and low cost, large chemical libraries looking for lead compounds [3]. Here, we describe the implementation in yeast and human cells of simple and innovative tools that can be used to screen for Rho-specific antibacterial compounds directly inside a eukaryotic cell. The tools are based on our previous work showing that the ectopic expression of $E$. coli Rho factor in the nucleus of $S$. cerevisiae induces a growth defect phenotype. We sought to extend the approach to screening conditions where any relevant inhibitor of Rho activity should suppress or alleviate the growth defect. The methodology was validated using the Rho-specific natural antibiotic BCM as a positive control.

The yeast tool was adapted to HTS-like conditions by monitoring cell growth and growth recovery in $200 \mu \mathrm{l}$ liquid cultures within 96-well plates in a semi-automated TECAN incubator. The presence of $\mathrm{BCM}$ in the culture medium mitigates the Rho-induced growth defect but did not lead to a complete suppression of the growth deficiency even at the concentration of $200 \mu \mathrm{g} / \mathrm{ml}$ which shows only a small improvement relative to $50 \mu \mathrm{g} / \mathrm{ml}$. At concentrations above $200 \mu \mathrm{g} / \mathrm{ml}$, the antibiotic became toxic to the yeast cells (results not shown). Presumably, the uptake of BCM is not optimal in yeast due to the presence of a cell wall. Nevertheless, the BCM-mediated mitigation of Rho-induced growth defect is clearly detectable within the beginning of the exponential phase of growth after 10 to $12 \mathrm{~h}$ of incubation. In the context of a fully automated and multiplexed HTS platform, such time frame should be appropriate to interrogate a large chemical library and reveal potential hits in a reasonable time and at low cost. Furthermore, the time necessary to reach the exponential phase can be shortened by changing the growth conditions such as raising the 
Fig. 7 Possible implication of a lower affinity of the ATP pocket in the moderate growth defect induced by Rho from $P$. aeruginosa. a Multiple sequence alignment showing the mutation Met-186-Ile within the ATP pocket of Rho from $P$. aeruginosa as compared to that of $E$. coli and other bacterial strains. b Rho protomer crystal structure PDB 1XPU [26] showing the ATP pocket where the adenine moiety of ATP is stacked between the side chains of the two conserved amino acid residues Met186 and Phe355
A
E. $\operatorname{col} 2$ S.enterica E.cloacae A.baumannis P. aeruginosa 167 186 214 PIGRGQRGLIVAPPKAGKTMLLQNIAQSIAYNHPDCVLMVLLIDERPE PIGRGQRGLIVAPPKAGKTMLLQNIAQS IAYNHPDCVLMVLLIDERPE PIGRGQRGLIVAPPKAGKTMLLQNIAQSIAYNHPDCVLMVLLIDERPE PIGKGQRSI IVAPPKAGKTMLLQNIAQSIVRNNPEVFLIVLLIDERPE PIGKGQRGLIVAPPKAGKTIMLQNIASNITRNNPECHLIVLLIDERPE

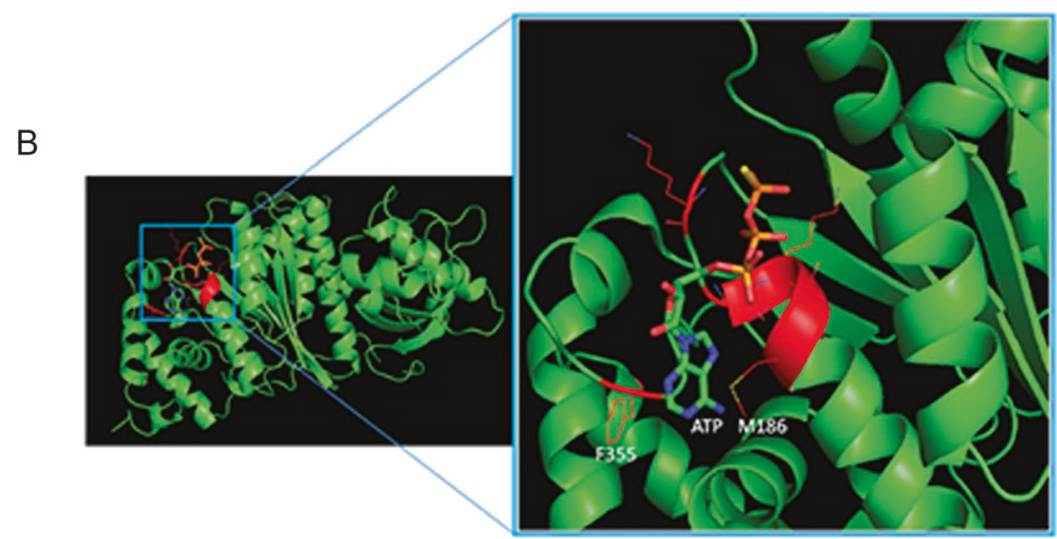

temperature of incubation to $30{ }^{\circ} \mathrm{C}$ instead of $25^{\circ} \mathrm{C}$ used in our experiments which should make the screening process less time-consuming. A subsequent analysis by the KirbyBauer diffusion test should be ideal to fine-tune the validation of potential hits regarding their specificity and possible toxicity at high concentration.

The extension of our approach to human cells provides additional strength to the screening tools by analyzing the effect of Rho inhibitors directly in the appropriate cellular environment which should exclude toxic compounds. Thus, despite a longer time frame for growth and detection of an effective inhibitor, the human cell line tool presents many advantages that should complement the yeast based assay. For instance, the cell line tool could be used to reevaluate hits selected by a first round of rapid screens made by the yeast tool. Indeed, with the human cell line tool, Rho inhibition by BCM induces a full relief of the growth defect which should give more confidence in the detection of positive hits. Also, the effect of BCM is observed at a relatively low concentration of the antibiotic, probably due to a higher uptake by the human cell in the absence of a cell wall. These features should increase the likelihood of discovering an efficient antibiotic by detecting numerous positive hits in a screening process. The positive hits will be subsequently submitted to downstream selections regarding major issues linked to bacterial strains such as uptake and avoidance by the efflux system.

Finally, we report that our screening tools can also be designed with Rho proteins originating from different bacterial pathogens. The use of a pathogen-defined tool in a screen may be helpful to discover drug candidates that inhibit Rho activity by binding to specific regions of the protein that are not shared with other bacterial strains. Interestingly, by testing the growth defect induced by the pathogen-defined tools we show that our approach can reveal subtle features regarding the functioning of a specific Rho factor. Thus, the tools could be utilized in studies connected with the structure/function relationship of Rho as a molecular motor.

Acknowledgements We are grateful to M Si-Tahar and V Hervé for discussions regarding the project and for sharing time slots to use the TECAN incubator. We thank R Washburn and K Schnetz for the gift of BCM. This work was supported by Région Centre Val-de-Loire through a research grant (Anti-Rho-Biotics) to A R Rahmouni and a $\mathrm{Ph} . \mathrm{D}$. fellowship to K Moreau.

\section{Compliance with ethical standards}

Conflict of interest The authors declare that they have no conflict of interests.

Open Access This article is licensed under a Creative Commons Attribution-NonCommercial-NoDerivatives 4.0 International License, which permits any non-commercial use, sharing, distribution and reproduction in any medium or format, as long as you give appropriate credit to the original author(s) and the source, and provide a link to the Creative Commons license. You do not have permission under this license to share adapted material derived from this article or parts of it. The images or other third party material in this article are included in the article's Creative Commons license, unless indicated otherwise in a credit line to the material. If material is not included in the article's Creative Commons license and your intended use is not permitted by statutory regulation or exceeds the permitted use, you will need to obtain permission directly from the copyright holder. To view a copy of this license, visit http://creativecommons.org/licenses/by-nc-nd/4.0/. 


\section{References}

1. Bush K, et al. Tackling antibiotic resistance. Nat Rev Microbiol. 2011;9:894-6.

2. Lukačišinová $M$, Bollenbach T. Toward a quantitative understanding of antibiotic resistance evolution. Curr Opin Biotechnol. 2017;46:90-7.

3. Brown ED, Wright GD. Antibacterial drug discovery in the resistance era. Nature. 2016;529:336-43.

4. Wambaugh MA, Shakya VPS, Lewis AJ, Mulvey MA, Brown JCS. High-throughput identification and rational design of synergistic small-molecule pairs for combating and bypassing antibiotic resistance. PLoS Biol. 2017;15: e2001644.

5. Peters JM, et al. Rho and NusG suppress pervasive antisense transcription in Escherichia coli. Genes Dev. 2012;26:2621-33.

6. Peters JM, et al. Rho directs widespread termination of intragenic and stable RNA transcription. Proc Natl Acad Sci USA. 2009;106:15406-11.

7. Bidnenko $\mathrm{V}$, et al. Termination factor Rho: from the control of pervasive transcription to cell fate determination in Bacillus subtilis. PLoS Genet. 2017;13:e1006909.

8. Cardinale CJ, et al. E. Termination factor Rho and its cofactors NusA and NusG silence foreign DNA in E. coli Science. 2008;320:935-8.

9. Krishna Leela J, Syeda AH, Anupama K, Gowrishankar J. Rhodependent transcription termination is essential to prevent excessive genome-wide R-loops in Escherichia coli Proc Natl Acad Sci USA. 2013;110:258-63.

10. Washburn RS, Gottesman ME. Transcription termination maintains chromosome integrity. Proc Natl Acad Sci USA. 2011;108: 792-7.

11. D'Heygere F, Rabhi M, Boudvillain M. Phyletic distribution and conservation of the bacterial transcription termination factor Rho. Microbiology. 2013;159:1423-36.

12. Williams RM, Durham CA. Bicyclomycin: synthetic, mechanistic, and biological studies. Chem Rev. 1988;88:511-40.

13. Zwiefka A, Kohn H, Widger WR. Transcription termination factor rho: the site of bicyclomycin inhibition in Escherichia coli. Biochemistry. 1993;32:3564-70.

14. Lawson MR, Dyer K, Berger JM. Ligand-induced and smallmolecule control of substrate loading in a hexameric helicase. Proc Natl Acad Sci USA. 2016;113:13714-9.

15. Skordalakes E, Brogan AP, Park BS, Kohn H, Berger JM. Structural mechanism of inhibition of the Rho transcription termination factor by the antibiotic bicyclomycin. Structure. 2005;13:99-109.

16. Botella. L, Vaubourgeix J, Livny J, Schnappinger D. Depleting Mycobacterium tuberculosis of the transcription termination factor Rho causes pervasive transcription and rapid death. Nat Commun. 2017;8:14731, https://doi.org/10.1038/ncomms14731.

17. Mosrin-Huaman C, Honorine R, Rahmouni AR. Expression of bacterial Rho factor in yeast identifies new factors involved in the functional interplay between transcription and mRNP biogenesis. Mol Cell Biol. 2009;29:4033-44.

18. Gari E, Piedrafita L, Aldea M, Herrero E. A set of vectors with a tetracycline-regulatable promoter system for modulated gene expression in Saccharomyces cerevisiae. Yeast. 1997;13: 837-48.

19. Kushnirov VV. Rapid and reliable protein extraction from yeast. Yeast. 2000;16:857-60.

20. Schwartz A, Margeat E, Rahmouni AR, Boudvillain M. Transcription termination factor rho can displace streptavidin from biotinylated RNA. J Biol Chem. 2007;282:31469-76.

21. Walmacq C, Rahmouni AR, Boudvillain M. Testing the steric exclusion model for hexameric helicases: substrate features that alter RNA-DNA unwinding by the transcription termination factor Rho. Biochemistry. 2006;45:5885-95.

22. Honorine R, Mosrin-Huaman C, Hervouet-Coste N, Libri D, Rahmouni AR. Nuclear mRNA quality control in yeast is mediated by Nrd1 co-transcriptional recruitment, as revealed by the targeting of Rho-induced aberrant transcripts. Nucleic Acids Res. 2011;39:2809-20.

23. Mosrin-Huaman C, Hervouet-Coste N, Rahmouni AR. Cotranscriptional degradation by the $5^{\prime}-3^{\prime}$ exonuclease Ratlp mediates quality control of HXK1 mRNP biogenesis in S. cerevisiae. RNA Biol. 2016;13:582-92.

24. Stuparevic I, Mosrin-Huaman C, Hervouet-Coste N, Remenaric $\mathrm{M}$, Rahmouni AR. Cotranscriptional recruitment of RNA exosome cofactors Rrp47p and Mpp6p and two distinct Trf-Air-Mtr4 polyadenylation (TRAMP) complexes assists the exonuclease Rrp6p in the targeting and degradation of an aberrant messenger ribonucleoprotein particle $(\mathrm{mRNP})$ in yeast. J Biol Chem. 2013;288:31816-29.

25. No D, Yao TP, Evans RM. Ecdysone-inducible gene expression in mammalian cells and transgenic mice. Proc Natl Acad Sci USA. 1996;93:3346-51.

26. Skordalakes E, Berger JM. Structural insights into RNAdependent ring closure and ATPase activation by the Rho termination factor. Cell. 2006;127:553-64. 